\title{
Intraoperative digital subtraction angiography in neurovascular disorders
}

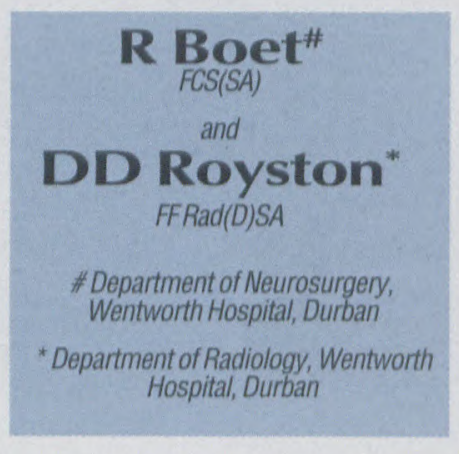

The importance of intraoperative DSA in the

management of complex

neurovascular disorders is explained. Both neurosurgeon and radiologist work in theatre as a team.

$\quad$ Abstract
Intraoperative digital subtraction
angiography is useful for
assessing the results of complex
neurovascular procedures. Fifty-
five patients with AVMs,
aneurysms (Berry and bacterial),
carotid-cavernous fistulae,
spontaneous intracranial
haemorrhages and penetrating
head injuries had intraoperative
angiograms. Sixteen of these
patients had findings on the
angiogram which altered the
surgical procedure. There were
no angiographic complications.
We found intraoperative digital
subtraction angiography a
valuable adjunct to several
neurovascular procedures.

\section{Introduction}

In the past neurosurgeons have relied mainly on direct visualisation or postoperative angiography to assess the results of complex neurovascular procedures. Advances in equipment have made it easier and faster to perform intraoperative angiography. Intraoperative angiography facilitates the immediate assessment of neurovascular procedures and allows the surgeon to correct any technical defects. We report our experience with intraoperative angiography atWentworth Hospital.

\section{Patients and methods}

From April 1990 to December 1994 fiftyfive intraoperative angiograms were performed at Wentworth Hospital. Angiograms were done via a transfemoral approach. A sheath was introduced preoperatively and flushed with heparinised saline $(2000 \mathrm{U} / 1000 \mathrm{ml}$ of normal saline at $30 \mathrm{ml} / \mathrm{hr}$ ).

The patient was then anaesthetised and placed in the required position for surgery. The theatre table had a radiolucent extension to facilitate screening of the aortic arch and neck vessels. The standard three-pin Mayfield-Kees head-holder was used as required.(Radiolucent carbon fibre headholders are available).

Angiography was performed with a mobile digital subtraction imaging system (Ziehm Exposcop CB7-D).This consisted of a $\mathrm{C}$-arm, digital processing unit, dual video monitors and an image storage unit.

The appropriate carotid or vertebral artery was catheterised during or at the end of the procedure as required by thesurgeon. Contrast Iohexol (6-10ml) (Omnipaque, Nycomed) was injected by hand to delineate the relevant vascular anatomy. The images were reviewed immediately. If it was felt that the surgical procedure was in any way unsatisfactory or incomplete further surgery was performed under the same 


\section{from page 24}

anaesthetic. The sheath was removed at the end of the procedure. Postoperatively the groin was monitored for the development of haematoma and peripheral pulses checked.

Routine postoperative angiography was not performed.

\section{Results}

Fifty-five intraoperative angiograms have been performed atWentworth Hospital. The surgical procedures performed are detailed in Table I. There were no angiographic complications.

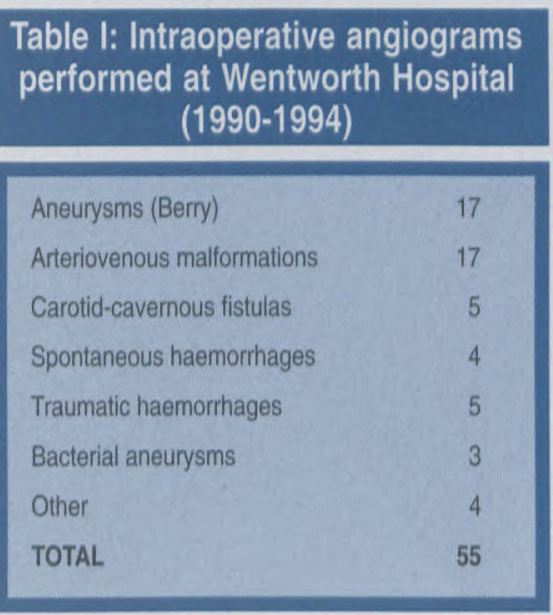

Although not strictly monitored the intraoperative angiogram added an additional forty-five to sixty minutes to the procedure (including the time required to place the femoral sheath preoperatively).

The four patients with spontaneous intracranial haemorrhage had intraoperative angiography, as their clinical condition necessitated immediate transfer to theatre for evacuation of the haematoma, precluding preoperative angiography. Intraoperative angiograms were used to localise pseudoaneurysms or arteriovenous fistulae in patients with traumatic haemorrhages following penetrating head injury. Patients with mycotic aneurysms had intraoperative angiograms to help localise the aneurysms. Intraoperative angiograms were used to monitor the successful surgical closure of carotid cavernous fistulae.

\section{Table Il: Cases with angiographic findings which altered the surgical procedure}

\begin{tabular}{|c|c|c|}
\hline Patient & Pathology & $\begin{array}{l}\text { Angiographic findings and } \\
\text { surgical action }\end{array}$ \\
\hline 4 & RPCOAA & Residual neck-clip repositioned \\
\hline 8 & LParietal AVM & Residual feeder-removed \\
\hline 11 & R Occipital AVM & Residual feeder \\
\hline 12 & Spontaneous ASDH & $\begin{array}{l}\text { Intraop angio demonstrated } \\
\text { the cause of ASDH-MCAA- clipped }\end{array}$ \\
\hline 15 & R Occipitoparietal AVM & Residual feeder-removed \\
\hline 16 & LCCF & $\begin{array}{l}\text { Residual CCF after packing- } \\
\text { repacked three times }\end{array}$ \\
\hline 17 & $R C C F$ & Residual CCF after packing-repacked \\
\hline 25 & $\begin{array}{l}\text { Transected vessel } \\
\text { following stab }\end{array}$ & $\begin{array}{l}\text { Angio showed transected } \\
\text { vessel which was then localised and clipped }\end{array}$ \\
\hline 27 & LOccipital AVM & Three intraop angiograms done until complete excision \\
\hline 34 & Bacterial aneurysms(3) & 4th Aneurysm identified with intraop angio \\
\hline 35 & R Occipital AVM & Residual feeder-removed \\
\hline 39 & Inflammatory MCAA & Spontaneous thrombosis of aneurysm shown on intraop angic \\
\hline 40 & Spontaneous ICH & Intraop angio demonstrated the cause of ICH-MCAA- clipped \\
\hline 50 & RICA Aneurysm & Non-filling of ICA following clipping - clip repositioned \\
\hline 53 & R Paraclinoid Aneurysm & Non filling of PCOA following clipping - clip repositioned \\
\hline 55 & LFrontal AVM & Residual AVM- removed \\
\hline \multicolumn{3}{|c|}{$\mathrm{PCOAA}$ - posterior communicating artery aneurysm } \\
\hline \multicolumn{3}{|c|}{ AVM - arteriovenous malformation } \\
\hline \multicolumn{3}{|c|}{ ASDH - acute subdural haematoma } \\
\hline \multicolumn{3}{|c|}{ MCAA - middle cerebral artery aneurysm } \\
\hline \multicolumn{3}{|c|}{ CCF - Carotid-cavernous fistula } \\
\hline \multicolumn{3}{|c|}{ ICA - Internal carotid artery } \\
\hline
\end{tabular}

Sixteen of these angiograms revealed findings which altered the surgical procedure (TableII). Six of the patients with AVMs required further surgery to completely excise the AVM. Three of the aneurysms had to have clips repositioned, due to a residual neck in one case and occluded vessels in two cases. Onemycotic aneurysm had thrombosed at the time of surgery. In a second patient with multiple mycotic aneurysms an additional aneurysm was detected on the intraoperative angiogram. Two patients with spontaneous intracerebral haemorrhages were found to have middle cerebral artery aneurysms. This finding enabled immediate clipping. In one of our patients a clip which was compromising the ICA was repositioned immediately due to the intraoperative angiogram finding.

\section{Discussion}

In the past, most intraoperative angiography was performed by using fluoroscopy or rapid serial-film angiography, following either direct puncture of the internal carotid or by retrograde catheterisation of the superficial temporal artery. ${ }^{1}$ Several authors have reported the use of a mobile digital subtraction imaging system. ${ }^{1,23}$ The advantages of this system are immediate review of subtracted images, high contrast resolution and superior spatial resolution (although not as good as conventional film 